\title{
APPEARANCE OF DUST IN FRAGMENTATIONS
}

\author{
BÉNÉDICTE HAAS *
}

\begin{abstract}
For fragmentations in which particles split even faster when their mass is smaller, it is possible to observe a decrease of the total mass of the system, due to the reduction into dust. We investigate here this appearance of dust for a large class of deterministic and random fragmentations.
\end{abstract}

Key words. Fragmentation, loss of mass to dust, tagged particle, subordinator.

AMS subject classifications. 60J25, 60J30, 82C21.

\section{Introduction}

In some particle fragmentation systems, one may observe a decrease of the total mass, although no mass is lost during each individual splitting. This is due to an intensive fragmentation which reduces positive-mass particles to an infinite number of zero-mass particles, or dust, in finite time. The lost mass is then lost to dust. This phenomenon may be seen as the dual process of gelation in coagulating systems, which corresponds to the appearance in finite time of an infinite-mass cluster (see [1], [13], [16]).

Our purpose is to study the appearance of dust in some fragmentation models that we now roughly describe. In these models, each particle is characterized by its mass, so that, starting from a particle with mass one, the natural space for our study is

$$
\mathcal{S}^{\downarrow}:=\left\{s=\left(s_{i}\right)_{i \in \mathbb{N}^{*}}, s_{1} \geq s_{2} \geq \ldots \geq 0: \sum_{i=1}^{\infty} s_{i} \leq 1\right\} .
$$

A sequence $s$ of $\mathcal{S}^{\downarrow}$ may be thought as the ranked rearrangement of masses of particles present at time $t$ and the difference $1-\sum_{i} s_{i}$ therefore corresponds to the mass of dust at that time. Informally, fragmentation systems are characterized by a family of break-up rates $\nu_{x}(d s), x>0$, that measure the speed at which particles with mass $x$ split in particles with masses $x s, s \in \mathcal{S}^{\downarrow}$. We consider here fragmentations with break-up rates that factorize as $\nu_{x}(d s)=\tau(x) \nu(d s)$ for some positive and continuous function $\tau$ on $] 0,1]$ and some measure $\nu$ on $\mathcal{S}^{\downarrow}$ such that $\nu\left(\sum_{i} s_{i}<1\right)=0$. This informal description will be made rigorous in Section 2 where both deterministic and random $(\tau, \nu)$-fragmentations are introduced.

Intuitively, when $\tau(x)$ increases sufficiently fast as $x \rightarrow 0$, the fragmentation may run away and create some dust. In Section 3, we characterize in terms of $\tau$ and $\nu$ when some mass is lost to dust and when all the mass is reduced to dust in finite time, and we see that it is essentially the behavior of $\tau$ near 0 that determines the appearance of dust. The main idea of the proof consists in following a specific particle in the fragmentation (a particle "tagged at random"). Section 4 is devoted to the dust mass asymptotic behaviors (for small and large times) in the deterministic setting and to the asymptotic behavior of the distribution tail of the first time of total disintegration to dust, in the random setting.

Most of the results presented here are part of the paper [11] and we refer to it for technical details. We also mention the papers [9], [8], [15], [6], [10], and [13] for

*Laboratoire de Probabilités et Modèles Aléatoires; Université Pierre et Marie Curie, et C.N.R.S. UMR 7599, 175, rue du Chevaleret, F-75013 Paris, France (haas@ccr.jussieu.fr). 
discussions on the appearance of dust for some different classes of deterministic and random fragmentations.

\section{Fragmentation models}

Let $\tau$ be a positive continuous function on $] 0,1]$ such that $\tau(1)=1$ and $\nu$ a non-negative measure on $\mathcal{S}^{\downarrow} \backslash\{(1,0,0, \ldots)\}$ such that

$$
\int_{\mathcal{S} \downarrow}\left(1-s_{1}\right) \nu(d s)<\infty .
$$

Such a measure $\nu$ is called a dislocation measure and may be seen as the break-up rate of a particle with mass one. Here we assume moreover that $\nu\left(\sum_{i} s_{i}<1\right)=0$, which means that no mass is lost at each separate dislocation, and we exclude the trivial case $\nu=0$. We now introduce two $(\tau, \nu)$-models - one deterministic and the other stochastic - to describe fragmentation systems in which a particle with mass $x$ breaks up in particles with masses $x s, s \in \mathcal{S}^{\downarrow}$, at a rate $\tau(x) \nu(d s)$. Note that in such models, particles may split in an infinite number of fragments and that $\nu$ may be infinite.

Deterministic model: it is described by a family of PDE's depending on the rate $\tau(x) \nu(d s)$. These PDE's modelize the evolution of $\left(\mu_{t}, t \geq 0\right)$, a family of nonnegative measures on $] 0,1]$, where $\mu_{t}(d x)$ represents the average number per unit volume of particles with mass in the interval $[x, x+d x[$ at time $t$ :

$$
\left\{\begin{array}{l}
\partial_{t}\left\langle\mu_{t}, f\right\rangle=\int_{0}^{1} \tau(x) \int_{\mathcal{S} \downarrow}\left[\sum_{i=1}^{\infty} f\left(x s_{i}\right)-f(x)\right] \nu(d s) \mu_{t}(d x), \quad t \geq 0, \\
\mu_{0}=\delta_{1}
\end{array}\right.
$$

the test-functions $f$ being differentiable functions with compact support in $] 0,1]$. This family of equations admits a unique solution (see Theorem 1 below). The first term in the integral describes the appearance of particles with masses $x s, s \in \mathcal{S}^{\downarrow}$, as a consequence of the dislocation of particles with mass $x$, and the second term corresponds to the disappearance of the particles with mass $x$ that have broken up. That $\mu_{0}=\delta_{1}$ means that there are only particles with mass one at the initial time.

Random model: this model is closely related to the homogeneous and selfsimilar fragmentation processes introduced by Bertoin in [4] and [5]. For a $\mathcal{S} \downarrow$-valued Markov process $X$, let $P_{r}$ denote the law of $X$ starting from $(r, 0, \ldots)$. A fragmentation process is a $\mathcal{S}^{\downarrow}$-valued Markov process $X$, such that for all $t, t^{\prime} \geq 0$, conditionally on $X(t)=\left(s_{1}, s_{2}, \ldots\right) \in \mathcal{S}^{\downarrow}, X\left(t+t^{\prime}\right)$ has the same law as the decreasing rearrangement of components of sequences $X^{(1)}\left(t^{\prime}\right), X^{(2)}\left(t^{\prime}\right), \ldots$ where the $X^{(i)}$ 's are independent processes with respective law $P_{s_{1}}, P_{s_{2}}, \ldots$. A fragmentation process is said to be homogeneous if for all $r \in] 0,1]$, the law of $X$ under $P_{r}$ is the same as that of $r X$ under $P_{1}$. Bertoin [4] and Berestycki [2] show that the laws of homogeneous fragmentation processes are characterized by a pair $(c, v)$, where $c$ is a nonnegative real number and $\nu$ a dislocation measure. The coefficient $c$ corresponds to a deterministic erosion phenomenon, that we do not consider here, that is we focus on fragmentation processes with erosion coefficient $c=0$. For dislocation measure such that $\nu\left(\sum_{i} s_{i}<1\right)=0$, the $(0, \nu)$-homogeneous fragmentation process will be our $(1, \nu)$-random model (i.e. $\tau(x)=1, x>0$ ) and we denote it by $X^{1, \nu}$. Note that the fragmentations rates of such models do not depend on the masses of the particles. 
For functions $\tau \neq 1$, the expected $(\tau, \nu)$ random model is a fragmentation process, that we denote $X^{\tau, \nu}$, constructed from $X^{1, \nu}$ by using time-changes depending on $\tau$. Let us present this construction. As proved in [5], there exists a family $\left(F^{1, \nu}(t), t \geq 0\right)$ of nested random open sets of ]0,1[ such that $X^{1, \nu}(t)$ is the decreasing sequence of the lengths of the interval components of $F^{\tau, \nu}(t)$, for all $t \geq 0$. For $x \in F^{1, \nu}(t)$, let $I_{x}^{1, \nu}(t)$ denote the interval of $F^{1, \nu}(t)$ that contains $x$. If $x \notin F^{1, \nu}(t)$, set $I_{x}^{1, \nu}(t):=\emptyset$. Writing $|I|$ for the length of an interval $I$, consider then for every $x \in] 0,1[$ and $t \geq 0$ the time-change

$$
T_{x}^{\tau, \nu}(t):=\inf \left\{u \geq 0: \int_{0}^{u} \frac{d r}{\tau\left(\left|I_{x}^{1, \nu}(r)\right|\right)}>t\right\}
$$

with the convention $\tau(0)=\infty$ and inf $\{\emptyset\}=\infty$. Define next for every $t \geq 0$ the open set

$$
F^{\tau, \nu}(t):=\bigcup_{x \in] 0,1[} I_{x}^{1, \nu}\left(T_{x}^{\tau, \nu}(t)\right)
$$

and remark that for $x, y \in] 0,1\left[\right.$, the intervals $I_{x}^{1, \nu}\left(T_{x}^{\tau, \nu}(t)\right)$ and $I_{y}^{1, \nu}\left(T_{y}^{\tau, \nu}(t)\right)$ are either equal or disjoint and that $F^{\tau, \nu}\left(t^{\prime}\right) \subset F^{\tau, \nu}(t)$ for $t \leq t^{\prime}$. The wanted $X^{\tau, \nu}(t)$ is then the decreasing rearrangement of the lengths of the interval's components of $F^{\tau, \nu}(t), t \geq 0$. To prove that $X^{\tau, \nu}$ is effectively a fragmentation process, one just has to adapt the proof of Theorem 2 in [5]. For $\tau(x)=x^{\alpha}, X^{\tau, \nu}$ is usually called a selfsimilar fragmentation process with index $\alpha$, since the law of $(X(t), t \geq 0)$ under $P_{r}$ is then the same as that of $\left(r X\left(r^{\alpha} t\right), t \geq 0\right)$ under $P_{1}$. We refer to [5] for background on these processes.

The deterministic and random $(\tau, \nu)$-fragmentations are related by the following theorem (cf. Th. 3 in [11])

THEOREM 2.1. The fragmentation equation (2.1) has a unique solution $\left(\mu_{t}^{\tau, \nu}, t \geq 0\right)$, which is given for all $t \geq 0$ by:

$$
\left\langle\mu_{t}^{\tau, \nu}, f\right\rangle=E\left[\sum_{i=1}^{\infty} f\left(X_{i}^{\tau, \nu}(t)\right)\right]
$$

for differentiable functions $f$ with compact support in $] 0,1]$.

The linearity of the fragmentation systems allows then us to express this solution $\mu_{t}^{\tau, \nu}$ as the hydrodynamic limit of some fragmentation processes. Indeed, consider $X^{\tau, \nu, 1}, \ldots, X^{\tau, \nu, n} n$ independent $(\tau, \nu)$-fragmentation processes and let $Y^{\tau, \nu, n}$ be the process obtained by reordering in the decreasing order, at each time $t$, the components of $X^{(1)}(t), \ldots, X^{(n)}(t)$. This process has the law of a fragmentation process starting from the sequence $(\underbrace{1,1, \ldots, 1}_{n \text { terms }}, 0, \ldots)$. Then, combining the previous result with the strong law of large number, we obtain that for each $t \geq 0$, with probability one,

$$
\frac{1}{n} \sum_{i=1}^{\infty} \delta_{Y_{i}^{\tau, \nu, n}(t)}(d x) \underset{\substack{\text { vaguely on }] 0,1] \\ n \rightarrow \infty}}{\longrightarrow} \mu_{t}^{\tau, \nu}
$$




\section{Appearance of dust}

The appearance of dust was first noted by Filippov for $(\tau, \nu)$-fragmentation equations with finite $\nu$. In Corollary 3 below we recover his results in that special case. In particular, when $\tau(x)=x^{\alpha}$, some dust appears if and only if $\alpha<0$. This last result was found independently and for some particular functions $\nu$ by some physicists [8], [15] in the 80's (they called the loss of mass to dust phenomenon "shattering"). More recently, Jeon [14] and Fournier and Giet [10] investigated the problem for fragmentation equations, which, restricted to our context, correspond to the case where $\nu$ is binary (i.e. $\left.\nu\left(s_{3}>0\right)=0\right)$ and $\nu\left(s_{1} \in d x\right)$ has a Lebesgue density. They also obtained that some mass is reduced to dust when $\tau(x)=x^{\alpha}$ with $\alpha<0$ and Fournier and Giet proved that this still occurs in systems where particles may moreover coagulate, provided that the coagulation rate is not too large. Both papers give some sufficient conditions for the appearance of dust in a somewhat more general setting, since their fragmentation rates are not assumed to factorize as here. Concerning $(\tau, \nu)$-fragmentation processes with $\tau(x)=x^{\alpha}$, Bertoin [6] showed that when $\alpha \geq 0$, almost surely no mass is reduced to dust, and that when $\alpha<0$, a.s. all the initial mass is disintegrated to dust in finite time.

Here, our purpose is to characterize the appearance of dust in terms of $\tau$ and $\nu$ in both deterministic and random models. In the random models, we also investigate the total disintegration to dust in finite time. We use an example to prove that in general, contrary to what occurs in the self-similar case $\left(\tau(x)=x^{\alpha}\right)$, appearance of dust does not imply total disintegration to dust.

3.1. Necessary and sufficient conditions for appearance of dust. In the deterministic setting, the total mass of positive-mass particles present at time $t$ is given by

$$
m^{\tau, \nu}(t)=\int_{0}^{1} x \mu_{t}^{\tau, \nu}(d x)
$$

and in the random setting by

$$
M^{\tau, \nu}(t)=\sum_{i} X_{i}^{\tau, \nu}(t)
$$

At time 0 , both masses are equal to 1 and some mass is lost to dust in the deterministic (resp. random) model if for some time $t, m^{\tau, \nu}(t)<1$ (resp. $\left.M^{\tau, \nu}(t)<1\right)$. By Theorem $1, m^{\tau, \nu}(t)=E\left[M^{\tau, \nu}(t)\right]$, which shows that $m^{\tau, \nu}(t)<1$ if and only if $M^{\tau, \nu}(t)<1$ with a positive probability. Actually, one may show that $P\left(\exists t: M^{\tau, \nu}(t)<1\right)$ is either 0 or 1 and then that some dust appears in the deterministic fragmentation if and only if some dust appears a.s. in the random one (see Th. 2 below).

To study this appearance of dust, we follow in the random model $X^{\tau, \nu}$ a particle tagged at random as follows. Consider $F^{\tau, \nu}$, the "interval representation" of $X^{\tau, \nu}$ introduced in Section 2, and let $U$ be a random variable uniformly distributed on $] 0,1[$ and independent of $F^{\tau, \nu}$. For every time $t \geq 0$, call $\lambda^{\tau, \nu}(t)$ the length of the interval components of $F^{\tau, \nu}(t)$ containing $U$, if such an interval exists, and set $\lambda^{\tau, \nu}(t):=0$ otherwise. The family of lengths $\left(\lambda^{\tau, \nu}(t), t \geq 0\right)$ represents then the masses of the tagged particle as time passes. The process $\lambda^{\tau, \nu}$ is obviously decreasing and may reach 0 in finite time. This first time at which $\lambda^{\tau, \nu}$ reaches 0 is the first time at which the tagged particle is reduced to dust. We denote that time by $T^{\tau, \nu}$, that is

$$
T^{\tau, \nu}:=\inf \left\{t \geq 0: \lambda^{\tau, \nu}(t)=0\right\} .
$$


This time can be infinite and that $T^{\tau, \nu}<\infty$ is intuitively connected to the appearance of dust. We now specify this. The process $\lambda^{\tau, \nu}$ has been constructed so that

$$
P\left(\lambda^{\tau, \nu}(t)>0\right)=P\left(U \in F^{\tau, \nu}(t)\right)=E\left[\sum_{i} X_{i}^{\tau, \nu}(t)\right]=m^{\tau, \nu}(t),
$$

since the open set $F^{\tau, \nu}(t)$ has total length $\sum_{i} X_{i}^{\tau, \nu}(t)$. Hence, $m^{\tau, \nu}(t)=P\left(T^{\tau, \nu}>t\right)$ and the existence of a time $t$ such that $m^{\tau, \nu}(t)<1$ is equivalent to $P\left(T^{\tau, \nu}<\infty\right)>$ 0 . To see when the latter happens, we use the following result, which is an easy consequence of Theorem 3 in [4]:

$$
\lambda^{\tau, \nu} \stackrel{\operatorname{law}}{=} \exp \left(-\xi_{\rho^{\tau, \nu}(.)}^{\nu}\right)
$$

where $\xi^{\nu}$ is a subordinator with Laplace exponent $\phi^{\nu}$ given for all $q \geq 0$ by

$$
\phi^{\nu}(q):=\int_{\mathcal{S} \downarrow}\left(1-\sum_{i=1}^{\infty} s_{i}^{q+1}\right) \nu(d s),
$$

and $\rho^{\tau, \nu}$ the time-change

$$
\rho^{\tau, \nu}(t)=\inf \left\{u \geq 0: \int_{0}^{u} d r / \tau\left(\exp \left(-\xi_{r}^{\nu}\right)\right)>t\right\}, t \geq 0 .
$$

We recall that a subordinator is a non-decreasing process starting from 0 with independent and stationary increments, that it is characterized by its Laplace exponent and that $E\left[e^{-q \xi_{r}^{\nu}}\right]=e^{-r \phi^{\nu}(q)}$, for all $r, q \geq 0$. We refer to [3] for background on the subject. All this shows that $T^{\tau, \nu} \stackrel{\operatorname{law}}{=} \int_{0}^{\infty} d r / \tau\left(\exp \left(-\xi_{r}^{\nu}\right)\right)$ and then that

$$
m^{\tau, \nu}(t)=P\left(\int_{0}^{\infty} d r / \tau\left(\exp \left(-\xi_{r}^{\nu}\right)\right)>t\right)
$$

One has then:

THEOREM 3.1. (i) The probability that some dust appears in the random $(\tau, \nu)$ fragmentation is either 0 or 1 and it is 1 if and only if some dust appears in the deterministic $(\tau, \nu)$-fragmentation.

(ii) For functions $\tau$ non-increasing near 0, some dust appears in the $(\tau, \nu)$-fragmentations if and only if

$$
\int_{0^{+}} \frac{\left(\phi^{\nu}\right)^{\prime}(x)}{\tau(\exp (-1 / x))\left(\phi^{\nu}\right)^{2}(x)} d x<\infty
$$

Proof. By (3.2), the existence of a time $t$ such that $m^{\tau, \nu}(t)<1$ is equivalent to $P\left(\int_{0}^{\infty} d r / \tau\left(\exp \left(-\xi_{r}\right)\right)<\infty\right)>0$. Lemma 3.6 in [3] states some results on the convergence of such integrals depending on a subordinator and one point is that the previous probability is either 0 or 1 when $\tau$ is non-increasing. This extends to general functions $\tau$ (see Prop. 10 in [11]) and then, since $m^{\tau, \nu}(t)=E\left[\sum_{i} X_{i}^{\tau, \nu}(t)\right]=$ $P\left(\int_{0}^{\infty} d r / \tau\left(\exp \left(-\xi_{r}\right)\right)>t\right)$, the existence of a time $t$ such that $m^{\tau, \nu}(t)<1$ is equivalent to the a.s. existence of time $t^{\prime}$ such that $M^{\tau, \nu}\left(t^{\prime}\right)<1$, which proves (i). The second part of the proof relies also on Lemma 3.6 in [3] and we refer to the proof of Prop. 6 in [11] for details. 
Some comments on this result. First, in order to simplify the statement we have considered functions $\tau$ non-increasing near 0 . But it is easy to see that if $\tau_{1} \leq \tau_{2}$, then $M^{\tau_{2}, \nu} \leq M^{\tau_{1}, \nu}$ (or equivalently $m^{\tau_{2}, \nu} \leq m^{\tau_{1}, \nu}$ ), so that some dust appears in the $\left(\tau_{2}, \nu\right)$-fragmentation as soon as it does in the $\left(\tau_{1}, \nu\right)$ 's one. Consequently, Theorem 2 gives sufficient (resp. necessary) conditions for the appearance of dust in a general $(\tau, \nu)$-fragmentation by considering some functions non-increasing near 0 smaller (resp. larger) than $\tau$.

As a Laplace exponent of a subordinator, $\phi^{\nu}$ is infinitely differentiable on $] 0, \infty[$ and concave. The derivative $\left.\left.\left(\phi^{\nu}\right)^{\prime}\left(0^{+}\right) \in\right] 0, \infty\right]$ (since the trivial case $\nu=0$ has been excluded) and we have then that $\left(\phi^{\nu}\right)^{2}(x) \geq c x^{2}\left(\phi^{\nu}\right)^{\prime}(x)$ for some positive constant $c$ and $x$ small enough. This gives with the previous theorem that for non-increasing near 0 functions $\tau$,

$$
\int_{0^{+}} d x / x \tau(x)<\infty \Rightarrow \text { appearance of dust }
$$

and we recover then that $\tau(x)=x^{\alpha}, \alpha<0$, (and more generally $\tau(x) \geq C|\ln x|^{\beta}$, $\beta>1$ ) is sufficient for the appearance of dust. Theorem 2 and an integration by parts show moreover that no mass is reduced to dust as soon as $\tau(x) \leq C x^{\alpha}, \alpha \geq 0$. When $\left(\phi^{\nu}\right)^{\prime}\left(0^{+}\right)<\infty$, we have $\left(\phi^{\nu}\right)^{2}(x) \underset{x \rightarrow 0}{\sim} x^{2}\left(\phi^{\nu}\right)^{\prime}\left(0^{+}\right)\left(\phi^{\nu}\right)^{\prime}(x)$ and Theorem 2 yields

COROLlary 3.2. For $(\tau, \nu)$-fragmentations such that $\tau$ is non-increasing near 0 and $\left(\phi^{\nu}\right)^{\prime}\left(0^{+}\right)<\infty$, the appearance of dust is equivalent to

$$
\int_{0^{+}} d x / x \tau(x)<\infty
$$

This result was obtained by Filippov [9] in the special case where $\nu\left(\mathcal{S}^{\downarrow}\right)<\infty$.

3.2. Total disintegration to dust in finite time. As shown in [6], selfsimilar fragmentation processes with a negative index $\alpha$ are a.s. entirely disintegrated to dust in finite time. The question that arises then is whether this equivalence between appearance of dust and total disintegration to dust is still valid for general $(\tau, \nu)$-fragmentations. Note that this concerns only the random fragmentations, since the probability (3.2) is (strictly) positive for all $t \geq 0^{1}$. So let $\zeta^{\tau, \nu}$ be the first time at which the mass of the random fragmentation is entirely reduced to dust, i.e.

$$
\zeta^{\tau, \nu}:=\inf \left\{t \geq 0: X_{1}^{\tau, \nu}(t)=0\right\} .
$$

By Prop. 12 in [11], one has

Proposition 3.3. For functions $\tau$ non-increasing near 0

(i) $\int_{0^{+}} d x / x \tau(x)<\infty \Rightarrow P\left(\zeta^{\tau, \nu}<\infty\right)=1$

(ii) if $\nu$ integrates $\left|\log \left(s_{1}\right)\right|$, the probability $P\left(\zeta^{\tau, \nu}<\infty\right)$ is either 0 or 1 and the following equivalence holds

$$
\int_{0^{+}} d x / x \tau(x)<\infty \Leftrightarrow P\left(\zeta^{\tau, \nu}<\infty\right)=1
$$

\footnotetext{
${ }^{1}$ If $\int_{0}^{\infty} d r / \tau\left(\exp \left(-\xi_{r}^{\nu}\right)\right) \stackrel{a . s .}{\leq} t$ for some deterministic $t$, then $T_{1}:=\inf \left\{r: \xi_{r}^{\nu} \geq 1\right\}$ would be bounded by some deterministic constant, and then, for times $r$ large enough, $\xi_{r}^{\nu} \stackrel{a . s .}{\geq} 1$. For such times $r$, we would have then $\exp \left(-r \phi^{\nu}(q)\right)=E\left[\exp \left(-q \xi_{r}^{\nu}\right)\right] \leq \exp (-q)$ for all $q \geq 0$ and then, since $\phi^{\nu}(q) / q \underset{q \rightarrow \infty}{\rightarrow} 0$ by dominated convergence, $0=\lim _{q \rightarrow \infty} \frac{\phi^{\nu}(q)}{q} \geq \frac{1}{r}$. The contradiction follows.
} 
As above, we point out that for general functions $\tau$, this result leads to sufficient (resp. necessary) conditions for total disintegration to dust by comparing $\tau$ with smaller (resp. larger) functions that are non-increasing near 0 . Let us also mention that the assumption on $\nu$ in (ii) is not so restrictive: for instance it is satisfied for measures $\nu$ such that $\nu\left(s_{n+1}>0\right)=0$ for some integer $n$ (which means that when a particle splits it splits in at most $n$ fragments). Indeed, for such measures, $n s_{1} \geq 1 \nu$-a.s. and therefore there exists a constant $C_{n}$ depending on $n$ such that $\int_{\mathcal{S} \downarrow}\left|\log \left(s_{1}\right)\right|(d s) \leq C_{n} \int_{\mathcal{S} \downarrow}\left(1-s_{1}\right) \nu(d s)$, which is finite.

Using Proposition 4 (i) and Corollary 3, we obtain that when $\left(\phi^{\nu}\right)^{\prime}\left(0^{+}\right)<\infty$ and the function $\tau$ is non-increasing near 0 , there are only two alternatives for random fragmentations: either a.s. no mass is disintegrated to dust or a.s. all the mass is disintegrated to dust in finite time. However, there are some $(\tau, \nu)$-fragmentations for which this equivalence between appearance of dust and total disintegration to dust does not hold, in the sense that $P\left(\exists t: M^{\tau, \nu}(t)<1\right)=1$ and $P\left(\zeta^{\tau, \nu}<\infty\right)=0$. See e.g. the example given in Section 5.2 of [11].

\section{Asymptotic behaviors}

In this section, we consider functions $\tau$ such that

$$
\left.C_{\beta} x^{-\beta} \leq \tau(x) \leq C_{\alpha} x^{-\alpha}, x \in\right] 0,1[
$$

for some fixed $\alpha \geq \beta>0, C_{\beta}, C_{\alpha}>0$. For such functions, we know that $(\tau, \nu)$ fragmentations lose mass to dust and that in the random models, all the mass is a.s. lost in finite time. The mass of dust at time $t$ is $1-m^{\tau, \nu}(t)$ in the deterministic case and $1-M^{\tau, \nu}(t)$ in the random case. In this section, we investigate the asymptotic behavior of these dust masses as $t \rightarrow 0$. We also study the asymptotic behavior of $m^{\tau, \nu}(t)$ and $P\left(\zeta^{\tau, \nu}>t\right)$ as $t \rightarrow \infty, \zeta^{\tau, \nu}$ being the first time at which $M^{\tau, \nu}(t)$ reaches 0 .

4.1. Deterministic behaviors. The study of $m^{\tau, \nu}(t)$ as $t \rightarrow 0$ and $t \rightarrow \infty$ relies on formula (3.2) and a first remark is that

$$
m^{\alpha, \nu}\left(C_{\alpha} t\right) \leq m^{\tau, \nu}(t) \leq m^{\beta, \nu}\left(C_{\beta} t\right), t \geq 0,
$$

where $m^{\alpha, \nu}(t)=P\left(\int_{0}^{\infty} \exp \left(-\alpha \xi_{r}^{\nu}\right) d r>t\right)\left(m^{\beta, \nu}\right.$ being defined similarly, by replacing $\alpha$ by $\beta$ ). As the asymptotic behaviors of $m^{\tau, \nu}$ can be infered from $m^{\alpha, \nu}$ and $m^{\beta, \nu}$, we shall now work with the $m^{\alpha, \nu}, \alpha>0$.

For all $t>0, m^{\alpha, \nu}(t)<1$, which means that some mass is reduced to dust at arbitrary small time. To see this, suppose that $m^{\alpha, \nu}(t)=1$ for near- 0 times $t$ and let $t$ be such that $m^{\alpha, \nu}(t)=1$ and $m^{\alpha, \nu}(2 t)<1$. Since $m^{\alpha, \nu}(t)=1$, one gets

$$
\exp \left(-\alpha \xi_{t / 2}^{\nu}\right) \int_{0}^{\infty} \exp \left(-\alpha\left(\xi_{r+t / 2}^{\nu}-\xi_{t / 2}^{\nu}\right)\right) d r \geq t / 2 \text { a.s. }
$$

and by the independence and stationarity of $\xi^{\nu}$ 's increments, the integral above is independent of $\xi^{\nu}$ and has the same law as $\int_{0}^{\infty} \exp \left(-\alpha \xi_{r}^{\nu}\right) d r$. Using also that $m^{\alpha, \nu}(2 t)<1$ and $P\left(\exp \left(-\alpha \xi_{t / 2}^{\nu}\right)<1 / 4\right)>0$ (the latter holds since the jump process of $\xi^{\nu}$ is a 
Poisson Point Process with a non-trivial intensity), we obtain

$$
\begin{aligned}
0 & <P\left(\exp \left(-\alpha \xi_{t / 2}^{\nu}\right)<1 / 4, \int_{0}^{\infty} \exp \left(-\alpha\left(\xi_{r+t / 2}^{\nu}-\xi_{t / 2}^{\nu}\right)\right) d r \leq 2 t\right) \\
& \leq P\left(\exp \left(-\alpha \xi_{t / 2}^{\nu}\right) \int_{0}^{\infty} \exp \left(-\alpha\left(\xi_{r+t / 2}^{\nu}-\xi_{t / 2}^{\nu}\right)\right) d r<t / 2\right)=0
\end{aligned}
$$

The contradiction follows and so $m^{\alpha, \nu}(t)<1$ for all $t>0$. One result in Section 4.2.1 of [11] specifies the decrease of $m^{\alpha, \nu}$ near 0 : for all $\varepsilon>0$, there exists a finite constant $C_{\varepsilon}$ such that $1-m^{\alpha, \nu}(t) \leq C_{\varepsilon} t^{1-\varepsilon+\underline{p} / \alpha}$ for $t>0$. The parameter $p$ depends on $\nu$ and is defined as

$$
\underline{p}=\sup \left\{q>0: \int_{\mathcal{S} \downarrow}\left(1-\sum_{i=1}^{\infty} s_{i}^{1-q} 1_{\left\{s_{i}>0\right\}}\right) \nu(d s)>-\infty\right\} .
$$

Concerning the behavior of $m^{\alpha, \nu}(t)$ as $t \rightarrow \infty$, a first remark is that the exponential functional $\int_{0}^{\infty} \exp \left(-\alpha \xi_{r}^{\nu}\right) d r$ has exponential moments (see [7]) and so there exist positive finite constants $A$ and $B$ such that $m^{\alpha, \nu}(t) \leq A \exp (-B t)$ for $t \geq 0$. This behavior can be specified when the Laplace exponent $\phi^{\nu}$ "varies nearly regularly with a positive index $a$ ". This means that there exist a function $f$ and two positive constants $C_{1}$ and $C_{2}$ such that $C_{1} f \leq \phi^{\nu} \leq C_{2} f$ and

$$
\frac{f(\lambda x)}{f(x)} \underset{x \rightarrow \infty}{\rightarrow} \lambda^{a} \text { for all } \lambda>0 .
$$

By the concavity of $\left.\phi^{\nu}, x \in\right] 0, \infty\left[\mapsto x / \phi^{\nu}(x)\right.$ is an increasing function, and by dominated convergence, it tends to $\infty$ as $x \rightarrow \infty$. Let $\psi$ be its inverse. We have then, Proposition 4.1. For dislocations measures $\nu$ such that $\phi^{\nu}$ varies nearly regularly with a positive index, there exist two positive constant $C$ and $D$ such that

$$
\exp (-D \psi(t)) \leq m^{\alpha, \nu}(t) \leq \exp (-C \psi(t)) \text { for } t \text { large enough }
$$

For the proof, we refer to Proposition 11 in [11].

4.2. Stochastic behaviors. The first point we are interested in is to know if, as in the deterministic case, some dust appears immediately. It depends whether $\nu$ is finite or not. Indeed, by results on homogeneous fragmentations (see [4]), we know that for finite $\nu$ the first split occurs after an exponential time, whereas for infinite $\nu$, the "splitting times" are dense in $\mathbb{R}^{+}$. Consequently, $M^{\tau, \nu}(t)=1$ near 0 when $\nu$ is finite and one may show (see e.g. [12]) that $M^{\tau, \nu}$ decreases strictly when $\nu$ is infinite. By Fatou's lemma and the deterministic results, this decrease can be specified: for all $\varepsilon>0, \liminf _{t \rightarrow 0}\left(1-M^{\tau, \nu}(t)\right) / t^{1-\varepsilon+\underline{p} / \alpha}=0, \underline{p}$ being defined by $(4.1)$.

Secondly, we are interested in the behavior of $P\left(\zeta^{\tau, \nu}>t\right)$ as $t \rightarrow \infty$. We recall that $\zeta^{\tau, \nu}$ is the time at which $M^{\tau, \nu}$ reaches 0 and that it is a.s. finite. Combining properties of self-similar fragmentation processes with the behavior of $m^{\alpha, \nu}(t)$ as $t \rightarrow \infty$, we obtain (see Prop. 14 in [11]) that the probability $P\left(\zeta^{\tau, \nu}>t\right)$ has the same type of behavior as $m^{\tau, \nu}(t)$ as $t \rightarrow \infty$, that is

Proposition 4.2. (i) There exist two positive constants, $A^{\prime}, B^{\prime}$ such that $P\left(\zeta^{\tau, \nu}>\right.$ $t) \leq A^{\prime} \exp \left(-B^{\prime} t\right)$ for $t \geq 0$. 
(ii) If $\phi^{\nu}$ varies nearly regularly with a positive index, there are two positive constants $C^{\prime}$ and $D^{\prime}$ such that for $t$ large enough

$$
\exp \left(-D^{\prime} \psi(t)\right) \leq P\left(\zeta^{\tau, \nu}>t\right) \leq \exp \left(-C^{\prime} \psi(t)\right)
$$

where $\psi$ is the inverse of the bijection $t \in[1, \infty) \mapsto t / \phi(t) \in[1 / \phi(1), \infty)$.

At last, let us mention that this result can be used to prove that for all $t>0$, $P\left(\zeta^{\tau, \nu} \leq t\right)>0$ (see Lemma 3 in $\left.[12]\right)$.

\section{REFERENCES}

[1] D.J. Aldous, Deterministic and stochastic models for coalescence (aggregation and coagulation): a review of the mean-field theory for probabilists, Bernoulli, 5:3-48, 1999.

[2] J. Berestycki, Ranked fragmentations, ESAIM P\&S, 6:157-176, 2002.

[3] J. Bertoin, Subordinators: Examples and Applications, In Bernard, P. (Ed.), Lectures on Probability Theory and Statistics, Ecole d'été de probabilités de St-Flour XXVII:1-91, Lect. Notes in Maths., 1717, Springer, Berlin, 1999.

[4] J. Bertoin, Homogeneous fragmentation processes, Probab. Theory Relat. Fields, 121(3):301318, 2001.

[5] J. Bertoin, Self-similar fragmentations, Ann. Inst. Henri Poincaré 38:319-340, 2002a.

[6] J. Bertoin, The asymptotic behavior of fragmentation processes, J. Eur. Math. Soc., 5(4):395416, 2003.

[7] P. Carmona, F. Petit, and M. Yor, On the distribution and asymptotic results for exponential functionals of Lévy processes, In M. Yor(Ed.), Exponential functionals and principal values related to Brownian motion, 73-121, Biblioteca de la Revista Matematica IberoAmericana, 1997.

[8] B.F. Edwards, M. Cao, and H. Han, Rate equation and scaling for fragmentation with mass loss, Phys. Rev., A, (41):5755-5757, 1990.

[9] A.F. Filippov, On the distribution of the sizes of particles which undergo splitting, Theory Probab. Appl., 6:275-294, 1961.

[10] N. Fournier and J.S. Giet, On small particles in coagulation-fragmentation equations, J. Stat. Phys., 111(5):1299-1329, 2003.

[11] B. Haas, Loss of mass in deterministic and random fragmentations, Stoch. Proc. Applic., 106(2):245-277, 2002.

[12] B. Haas, Regularity of formation of dust in self-similar fragmentations, to appear in Ann. Inst. Henri Poincaré Probab. Stat., 2004.

[13] I. Jeon, Existence of gelling solutions for coagulation-fragmentation equations, Comm. Math. Phys., 194:541-567, 1998.

[14] I. Jeon, Stochastic fragmentation and some sufficient conditions for shattering transition, J. Korean Math. Soc., 39 n $^{\circ}$ 4:543-558., 2002.

[15] E. McGrady and R. Ziff, "Shattering" transition in fragmentation, Phys. Rev. Lett., 58:892$895,1987$.

[16] J.R. Norris, Clusters coagulation, Comm. Math. Phys, 209:407-435, 2000. 